\title{
Increased Circulating CXCL10 in Non-Segmental Vitiligo Concomitant with Autoimmune Thyroid Disease and Alopecia Areata
}

\author{
Li Zhang*, Xinya Xu*, Shujun Chen, Yuli Kang, Xiuxiu Wang, Chengfeng Zhang, Leihong Xiang \\ Department of Dermatology, Huashan Hospital, Fudan University, Shanghai, China
}

Background: Vitiligo is a common acquired pigmentary disease caused by destruction of epidermal melanocytes in underlying autoimmune response. Few studies have been focused on the role of chemokines in non-segmental vitiligo (NSV) concomitant with autoimmune thyroid disease (AITD) and alopecia areata (AA). Objective: The aim of this study was to determine the best serum biomarker for predictive role in the progression of vitiligo and to evaluate the influence of AA and/or AITD on vitiligo by using the biomarker. Methods: This prospective cohort study recruited 45 NSV patients: 14 without either AITD or AA, 12 with AITD, 11 with AA, and 8 with both AITD and AA. Serum levels of CXCL1, CXCL8, CXCL9, CXCL10, CXCL12, CXCL13, and CXCL16 were analyzed by ELISA. CXCR3 mRNA expression was detected on PBMCs by RT-PCR. Improvement was evaluated using repigmentation scales. Results: Serum CXCL10 levels, along with the expression of CXCR3 mRNA were higher in NSV patients with AITD or AA alone than in those without

Received November 20, 2018, Revised January 2, 2019, Accepted for publication January 26, 2019

Corresponding author: Leihong Xiang, Department of Dermatology, Huashan Hospital, Fudan University, 12 Wulumuqi Zhong Road, Shanghai 200040, China. Tel: 86-21-5288999, Fax: 86-21-52887782, E-mail: flora xiang@vip.163.com

ORCID: https://orcid.org/0000-0002-6999-4686

Chengfeng Zhang, Department of Dermatology, Huashan Hospital, Fudan University, 12 Wulumuqi Zhong Road, Shanghai 200040, China. Tel: 86-21-5288999, Fax: 86-21-52887782, E-mail: e3dangdang@hotmail.com ORCID: https://orcid.org/0000-0002-1302-5667

*These authors contributed equally to this work.

This is an Open Access article distributed under the terms of the Creative Commons Attribution Non-Commercial License (http://creativecommons. org/licenses/by-nc/4.0) which permits unrestricted non-commercial use, distribution, and reproduction in any medium, provided the original work is properly cited.

Copyright $\odot$ The Korean Dermatological Association and The Korean Society for Investigative Dermatology
AITD or AA. Moreover, serum CXCL10 levels, along with the expression of CXCR3 mRNA were higher in NSV patients with both AITD and AA than in those with AITD or AA alone. Poorer repigmentation was observed in NSV patients with both AA and AITD than in those with AA or AITD alone. Conclusion: CXCL10 could be a biomarker to predict the progression of NSV. Dermatologists should pay much attention to those NSV patients concomitant with AITD and/or AA, for comorbidity might lead to more active autoimmune reaction. (Ann Dermatol 31(4) 393 402, 2019)

\section{-Keywords-}

Alopecia areata, Autoimmunity, Chemokine CXCL10, Thyroid diseases, Vitiligo

\section{INTRODUCTION}

Vitiligo is an acquired chronic depigmenting disorder affecting $0.5 \%$ to $2 \%$ of the world's population ${ }^{1}$. It is an under-diagnosed, organ-specific, autoimmune disease of the skin caused by $\mathrm{T}$ cell-mediated cytotoxic attack on epidermal melanocytes ${ }^{2}$. Chemokines and their receptors are vital for the trafficking of T cells in vitiligo and other autoimmune diseases. Moreover, dysregulation of chemokines and their receptors might lead to immune system homeostasis and inflammation ${ }^{3,4}$.

Much attention was paid to the biomarker role of chemokines including CXCL1, CXCL8, CXCL9, CXCL10, and $\mathrm{CXCL}_{13} 3^{5-10}$ and other biomarkers, including S100B and HMGB1. Increased serum level of S100B was demonstrated in vitiligo patients with active depigmentation compared to vitiligo patients with stable disease ${ }^{11}$. Moreover, greater expression of HMGB1 was observed in vitiliginous 
skin than that in the uninvolved $\operatorname{skin}^{12}$. In recent years, there have been increasing studies focusing on the biomarker role of chemokines. In 2017, CXCL12 and CXCL16 were reported as new biomarkers in vitiligo ${ }^{13,14}$. However, up to date, the clinical studies available were limited by short follow up time. Moreover, few studies showed the difference of predictive power among CXCL1, CXCL8, CXCL9, CXCL10, CXCL12, CXCL13, and CXCL16 in the progression of vitiligo.

A number of clinical studies have reported that the comorbidity of other autoimmune diseases in patients with vitiligo, such as autoimmune thyroid disease (AITD), alopecia areata (AA), type 1 diabetes, psoriasis, and atopic dermatitis $^{15,16}$. A recent study of meta-analysis showed that in patients affected by vitiligo the prevalence of AITD was $14.3 \%$, while positivity to thyroid-specific antibodies was found in $20.8 \%$ of them ${ }^{17}$. Vitiligo and AA share a similar pathogenesis, as they are both interferon (IFN)- $\gamma$-driven and dependent on CD8 $+\mathrm{T}_{\text {cells }}{ }^{18}$. However, limited clinical data were available regarding chemokine levels in vitiligo and its associated autoimmune diseases AA and AITD.

Therefore, in this study, we aimed at determining the best serum biomarker from CXCL1, CXCL8, CXCL9, CXCL10, CXCL12, CXCL13, and CXCL16 for predictive role in the progression of vitiligo. Furthermore, in order to evaluate the influence of AA and/or AITD on vitiligo, we analyzed the progression of vitiligo in presence or absence of $A A$ and/ or AITD by using the biomarker.

\section{MATERIALS AND METHODS}

\section{Patients}

This prospective study was conducted at Huashan Hospital, Fudan University, China, from July 2016 to July 2018. All the participants signed a written informed consent form, in accordance with the standards of the Institutional Ethics Committee of Huashan Hospital, Fudan University (HIRB 2017M-006). Forty-five patients were recruited who had been independently diagnosed with progressive vitiligo by two dermatologists, and 15 healthy individuals were included as controls. All patients were diagnosed as non-segmental vitiligo (NSV) with a body surface area involvement over $15 \%$. Progressive vitiligo was defined in cases that had a Vitiligo European Task Force spreading score of +1 to $+5^{19}$. Among the 45 patients with progressive NSV, 14 did not have either AA or AITD, 11 had AA, 12 had AITD, 8 had both AA and AITD. Patients in either group were excluded if they had previously been treated using immunosuppressive therapy, such as corticosteroids, calcineurin inhibitors, or phototherapy. Data were collected regarding sex, age, disease duration, VASI score, signs and symptoms of autoimmune thyroid disorders and AA. The diagnostic criterion for AITD was having positive thyroid autoantibodies including thyroid-stimulating hormone receptor autoantibodies (TRAb), thyroid peroxides autoantibodies (TPOAb), and/or thyroglobulin autoantibodies, with either abnormal or normal thyroid function, including chronic autoimmune thyroiditis or Hashimoto's thyroiditis, painless thyroiditis, atrophic thyroiditis or primary hypothyroidism and Graves' disease ${ }^{20}$. Diagnosis of AA was made by dermatologists, clinically from the presence of circumscribed, hairless patches or large alopecic areas in otherwise normal-appearing skin areas. Totally, 19 patients were diagnosed as AA including 11 NSV patients with AA alone and 8 NSV patients with $\mathrm{AA}$ and AITD. In NSV patients with AA alone, the mean duration was $5 \pm 5.1$ months with the bald skin area of $18.5 \% \pm 4.8 \%$; while in NSV patients with AA and AITD, the mean duration was $5 \pm 4.4$ months with the bald skin area of $16.9 \% \pm 6.6 \%$. The patients were treated using 1-ml intramuscular injections of diprospan (containing 2 $\mathrm{mg}$ betamethasone sodium phosphate and $5 \mathrm{mg}$ betamethasone dipropionate) once per month until the progression of vitiligo had been arrested for a maximum of 6 injections. Topical tacrolimus $0.1 \%$ was applied twice daily to all spreading lesions. Topical dexamethasone was applied to patients with AA twice daily to the AA lesions. Narrowband ultraviolet B phototherapy twice a week for NSV since progression was arrested. Oral levothyroxine was applied to patients with AITD according to endocrinologist's suggestion. The samples of peripheral blood were collected at the first visit to our department, as well as at the third month, sixth month, ninth month, and twelfth month.

\section{Enzyme linked immunosorbent assay (ELISA) detection of chemokines}

Serum levels of CXCL1, CXCL8, CXCL9, CXCL10, CXCL12, CXCL13, and CXCL16 levels were assayed using an ELISA kit (R\&D Systems, USA).

\section{Real-time polymerase chain reaction detection of CXCR3 and expression}

Total RNA was extracted from peripheral blood mononuclear cells (PBMCs) using TRIzol (Invitrogen, Carlsbad, CA, USA). First-strand cDNA was then synthesized from DNAse-treated total RNA using an oligo-dT18 primer and RevertAid $^{\text {TM }}$ M-MLV reverse transcriptase. Transcripts were quantified by real-time quantitative polymerase chain reaction (PCR) on a 7500 Real-Time PCR System (Applied Biosystems, Minneapolis, MN, USA) using SYBR Premix 
Ex Taq II (TaKaRa, Ohtsu, Japan) according to the manufacturer's instructions. The primers for CXCR3 were 5'GCCGAGAAAGCAGGGTAGAC-3' (forward) and GCA AGAGCAGCATCCACAT-3' (reverse), while those for the $\beta$-actin endogenous control were $5^{\prime}$-CTCCATCCTGGCC TCGCTGT-3' (forward) and 5'-GCTGTCACCTTCACCG TTCC-3' (reverse). The PCRs were cycled 40 times after the initial denaturation step of $95^{\circ} \mathrm{C}$ for 30 seconds, with the following parameters: $95^{\circ} \mathrm{C}$ for 5 seconds and $60^{\circ} \mathrm{C}$ for 34 seconds. The relative fold change of mRNA expression was calculated using the $2^{-\Delta \Delta \mathrm{Ct}}$ method. The mRNA levels of the target gene were normalized to the levels of $\beta$-actin.

\section{Repigmentation measurement}

After 12 months treatment, repigmentation (defined as percentage reduction in involved skin area) from the baseline measurements was quantified by two independent dermatologists using the following visual scale: Grade 0, less than $1 \%$ improvement; Grade 1, $1 \%$ to $25 \%$ improvement; Grade 2, 26\% to $50 \%$ improvement; Grade 3, 51\% to $75 \%$ improvement; Grade 4, more than 75\% improvement.

\section{Statistical analysis}

Values were given as mean \pm standard deviation for normally distributed variables. Differences in variables were analyzed using the ANOVA, Student's t-, and $\chi^{2}$ tests as appropriate, and $p$-values less than 0.05 were considered significant. IBM SPSS ver. 19.0 Statistics software (IBM Corp., New York, NY, USA) was used for all analyses.

\section{RESULTS}

\section{Description of patients}

The clinical characteristics of patients are summarized in Table 1.

\section{Elevated serum levels of CXCL9, CXCL10, CXCL12, and CXCL16 in patients with vitiligo}

Serum levels of CXCL9 $(270.4 \pm 29.1 \mathrm{pg} / \mathrm{ml}$ in patients with vitiligo vs. $99.2 \pm 18.3 \mathrm{pg} / \mathrm{ml}$ in health controls, $p<0.001)$,
CXCL10 $(916.9 \pm 157.3 \mathrm{pg} / \mathrm{ml}$ in patients with vitiligo vs. $117.1 \pm 28.1 \mathrm{pg} / \mathrm{ml}$ in health controls, $p<0.001)$, CXCL12 $(1,797.0 \pm 181.8 \mathrm{pg} / \mathrm{ml}$ in patients with vitiligo vs. $1,163.0 \pm$ $140.0 \mathrm{pg} / \mathrm{ml}$ in health controls, $p<0.001)$ and CXCL16 $\left(1,718.0 \pm 368.6 \mathrm{pg} / \mathrm{ml}\right.$ in patients with vitiligo vs. $1,391.0_{ \pm}$ $213.1 \mathrm{pg} / \mathrm{ml}$ in health controls, $p<0.05$ ) were significantly higher in blood collected at baseline from patients with vitiligo than in that collected from healthy controls. However, serum levels of CXCL1 $(182.4 \pm 71.5 \mathrm{pg} / \mathrm{ml}$ in patients with vitiligo vs. $145.6 \pm 22.4 \mathrm{pg} / \mathrm{ml}$ in health controls, $p>0.05)$, CXCL8 $(131.8 \pm 50.2 \mathrm{pg} / \mathrm{ml}$ in patients with vitiligo vs. $97.5 \pm 29.5 \mathrm{pg} / \mathrm{ml}$ in health controls, $p>0.05)$ and CXCL13 $(29.8 \pm 4.8 \mathrm{pg} / \mathrm{ml}$ in patients with vitiligo vs. $25.7 \pm 5.2 \mathrm{pg} / \mathrm{ml}$ in health controls, $p>0.05$ ) did not differ significantly between patients with vitiligo and healthy controls (Fig. 1).

\section{Down-regulation of serum CXCL10 levels in response to treatment}

To assess the therapy effect of serum CXCL9, CXCL10, CXCL12, and CXCL16, serum levels of each were measured in patients with NSV after twelve months treatment. Successful treatment led to a decrease in serum CXCL10 levels $(916.9 \pm 157.3 \mathrm{pg} / \mathrm{ml}$ in patients before treatment vs. $543.8 \pm 82.3 \mathrm{pg} / \mathrm{ml}$ in patients after treatment, $p<0.0001)$. However, there were no significant changes in serum levels of CXCL9 $(270.4 \pm 29.1 \mathrm{pg} / \mathrm{ml}$ in patients before treatment vs. $264.5 \pm 35.9 \mathrm{pg} / \mathrm{ml}$ in patients after treatment, $p>0.05), C X C L 12(1,797.0 \pm 181.8 \mathrm{pg} / \mathrm{ml}$ in patients before treatment vs. $1,701.0 \pm 175.6 \mathrm{pg} / \mathrm{ml}$ in patients after treatment, $p>0.05)$ or CXCL16 $(1,718.0 \pm 368.6 \mathrm{pg} / \mathrm{ml}$ in patients before treatment vs. $1,709.0 \pm 382.3 \mathrm{pg} / \mathrm{ml}$ in patients after treatment, $p>0.05$; Fig. 2).

\section{Increased levels of serum CXCL10 predict progression in vitiligo}

To assess the predictive role of serum CXCL9, CXCL10, CXCL12, and CXCL16, serum levels of each were measured patients with NSV in the sixth month during the follow up. Higher serum CXCL10 levels were shown in patients with progression compared with those without pro-

Table 1. The characteristics of patients with NSV

\begin{tabular}{lccccrr}
\hline Characteristic & Healthy controls & $\begin{array}{c}\text { NSV without } \\
\text { AA and AITD }\end{array}$ & NSV with AA & NSV with AITD & $\begin{array}{c}\text { NSV with } \\
\text { AA and AITD }\end{array}$ & $p$-value \\
\hline Sex (female/total $n)$ & $7 / 8$ & $5 / 8$ & $5 / 6$ & $6 / 6$ & $4 / 4$ & 0.3063 \\
Age (yr) & $34 \pm 5.5$ & $33 \pm 7.1$ & $33 \pm 6.4$ & $32 \pm 7.1$ & $32 \pm 6.0$ & 0.9856 \\
Duration (mo) & $/$ & $5 \pm 5.9$ & $5 \pm 5.1$ & $5 \pm 4.9$ & $5 \pm 4.4$ & $>0.9999$ \\
VASI score & $/$ & $18 \pm 9.8$ & $18 \pm 10.4$ & $19 \pm 12.0$ & $19 \pm 3.0$ & 0.9997 \\
\hline
\end{tabular}

NSV: non-segmental vitiligo, AA: alopecia areata, AITD: autoimmune thyroid disease, VASI: vitiligo area severity index. $\chi^{2}$ test was applied. 
A

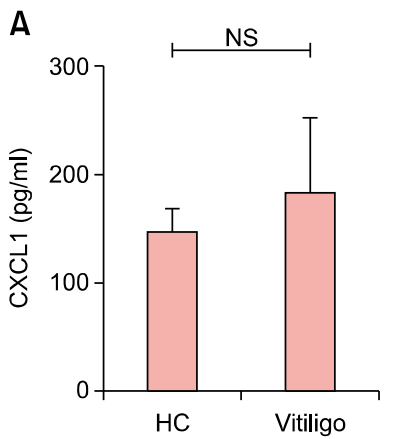

E

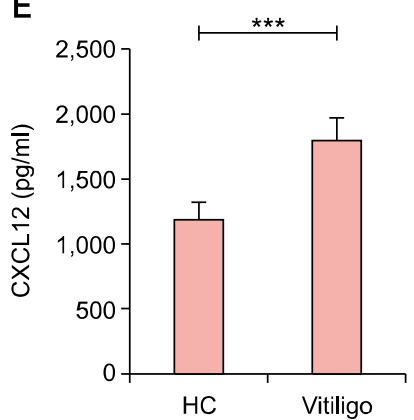

B

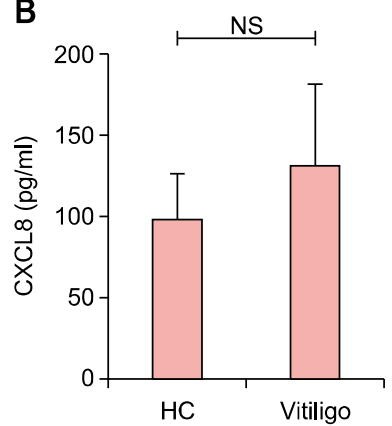

$\mathrm{F}$

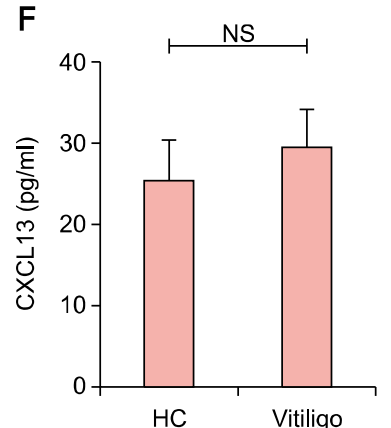

C

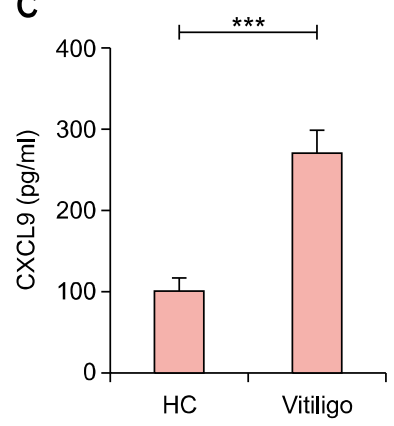

G

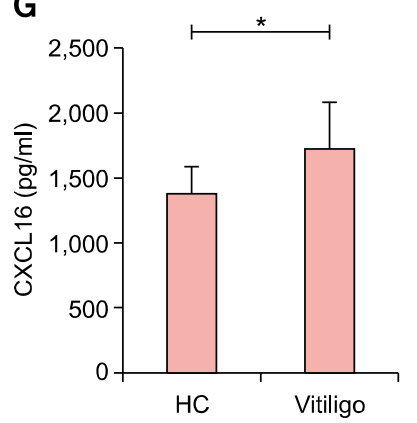

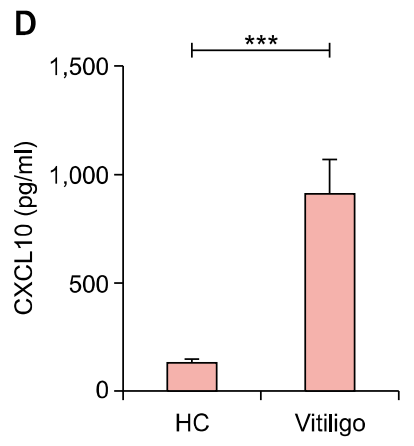

Fig. 1. Chemokines in the serum of patients with non-segmental vitiligo (NSV). (A) CXCL1, (B) CXCL8, (C) CXCL9, (D) CXCL10, (E) CXCL12, (F) CXCL13, and (G) CXCL16. Student's t-test was applied. The serum levels of CXCL9, CXCL10, CXCL12, and CXCL16 were higher in patients with NSV than in healthy controls $(\mathrm{HC})$. However, there was no significant difference in the serum levels of CXCL1, CXCL8, or CXCL13 between patients with NSV and healthy controls. NS: no significance. ${ }^{*} p<0.05,{ }^{* * *} p<0.001$.

A

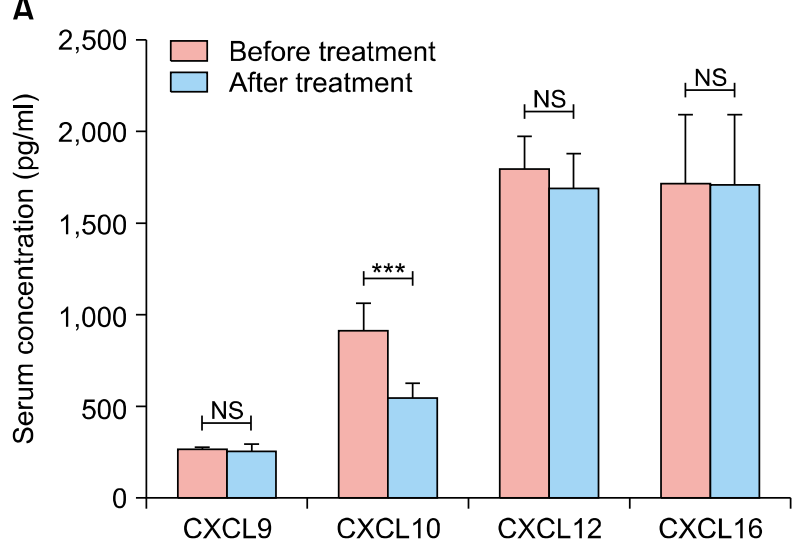

B

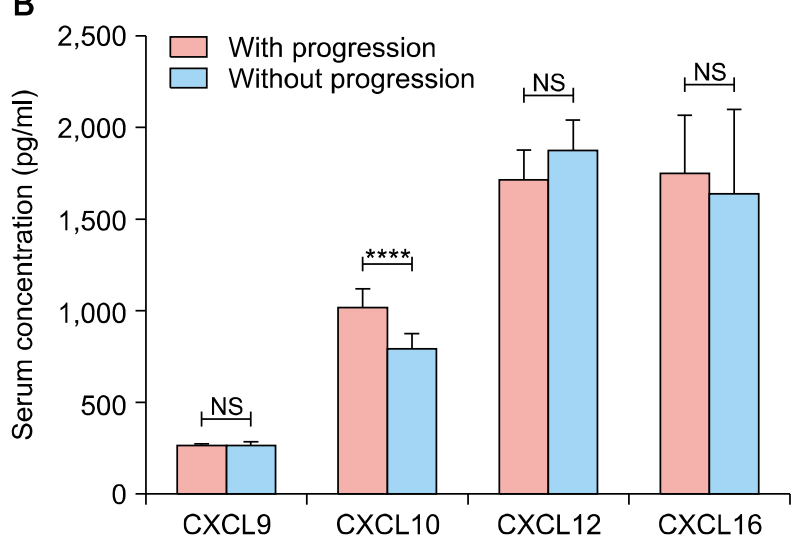

Fig. 2. Decreased chemokine levels after treatment of patients with non-segmental vitiligo (NSV). Student's t test was applied. Serum levels of CXCL10 had decreased significantly after treatment. However, those of CXCL9, CXCL12, and CXCL16 had not (A). Higher serum levels of CXCL10 in patients with vitiligo underwent progression in the following up (B). NS: no significance. ${ }^{* * *} p<0.001$, $* * * * p<0.0001$.

gression $(1,028.0 \pm 109.3 \mathrm{pg} / \mathrm{ml}$ in patients with progression vs. $799.4 \pm 79.1 \mathrm{pg} / \mathrm{ml}$ in patients without progression, $p<0.0001)$. However, there were no significant changes in serum levels of CXCL9 $(271.7 \pm 23.2 \mathrm{pg} / \mathrm{ml}$ in patients with progression vs. $269.2 \pm 36.4 \mathrm{pg} / \mathrm{ml}$ in patients without progression, $p>0.05), C X C L 12(1,727.0 \pm 168.2 \mathrm{pg} / \mathrm{ml}$ in patients with progression vs. $1,894.0 \pm 167.9 \mathrm{pg} / \mathrm{ml}$ in patients without progression, $p>0.05)$ or CXCL16 $\left(1,763.0_{ \pm}\right.$ $319.2 \mathrm{pg} / \mathrm{ml}$ in patients with progression vs. 1,654.0 \pm $460.5 \mathrm{pg} / \mathrm{ml}$ in patients without progression, $p>0.05$; Fig. 2) in patients with progression compared with those without progression. 


\section{Higher serum levels of CXCL10 in patients who had vitiligo with $A A$ and AITD}

The serum level of CXCL10 was $732.5 \pm 100.7 \mathrm{pg} / \mathrm{ml}$ in patients who had NSV without AA or AITD, 917.5 \pm 113.5 $\mathrm{pg} / \mathrm{ml}$ in patients who had NSV with $\mathrm{AA}, 916.3 \pm 61.6$ $\mathrm{pg} / \mathrm{ml}$ in patients who had NSV with AITD, and 1,094.0 \pm $109.1 \mathrm{pg} / \mathrm{ml}$ in patients who had NSV with AA and AITD. The levels of CXCL10 in patients who had NSV with AA were higher than those in patients who had NSV without AA or AITD ( $p<0.05$, Fig. 3). Similarly, the levels of CXCL10 in patients who had NSV with AITD were higher than those in patients who had NSV without AA or AITD $(p<$ 0.01, Fig. 3). However, the levels of CXCL10 did not differ between patients who had NSV with AA and those who had NSV with AITD ( $p>0.05$, Fig. 3). Moreover, the levels of CXCL10 in patients who had NSV with both AA and AITD were higher than those in patients who had NSV with AA, NSV with AITD, and NSV without either AA or AITD ( $p<0.05, p<0.05, p<0.001$, respectively; Fig. 3).

\section{Elevated expression of CXCR3 mRNA in the PBMCs of patients with vitiligo}

Since serum CXCL10 was significantly increased in patients with NSV, especially in those who also had AA and

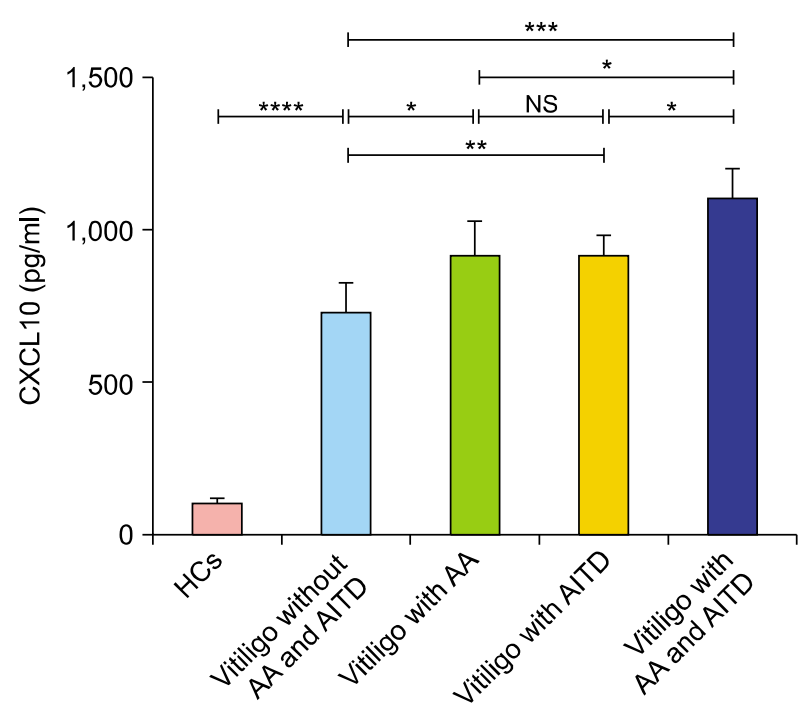

Fig. 3. Differences in serum CXCL10 levels in patients who had non-segmental vitiligo (NSV) with alopecia areata (AA), and/or autoimmune thyroid disease (AITD). ANOVA test was applied. The levels of CXCL10 in patients who had NSV with both AA and AITD were higher than in those who had NSV with AA or AITD alone. The levels of CXCL10 in patients who had NSV with either AA or AITD were higher than in those who had NSV without AA or AITD. HCs: healthy controls, NS: no significance. ${ }^{*} p<0.05,{ }^{* *} p<0.01,{ }^{* * *} p<0.001,{ }^{* * * *} p<0.0001$.
AITD, we hypothesized that CXCR3, the common receptor for CXCL10, might be up-regulated in the PBMCs of patients who had NSV with AA and/or AITD. Indeed, RT-PCR demonstrated that CXCR3 mRNA expression was higher in the PBMCs of patients who had NSV with both AA and AITD than in those who had NSV with either AA or AITD alone $(12.1 \pm 1.2$ in vitiligo patients with both $A A$ and AITD vs. $7.7 \pm 1.4$ in vitiligo patients with AA alone; $p<0.01$ and $12.1 \pm 1.2$ in vitiligo patients with both $A A$ and AITD vs. $7.7 \pm 2.2$ in vitiligo patients with AITD alone; $p<0.05$, respectively; Fig. 4). In addition, CXCR3 mRNA expression was higher in patients who had NSV with AA or AITD alone than in those who had NSV without either AA or AITD $(7.7 \pm 1.4$ in vitiligo patients with AA alone and $7.7 \pm 2.2$ in vitiligo patients with AITD alone vs. $4.9 \pm$ 0.7 in vitiligo patients without AA or AITD, respectively; $p<$ 0.01 in both cases; Fig. 4).

\section{The presence of AA and AITD in NSV leads to more active autoimmune reaction}

We next explored the changes of CXCL10 after 3, 6, 9, and 12 months of treatment in patients who had NSV with AA and/or AITD. The level of CXCL10 in patients who had

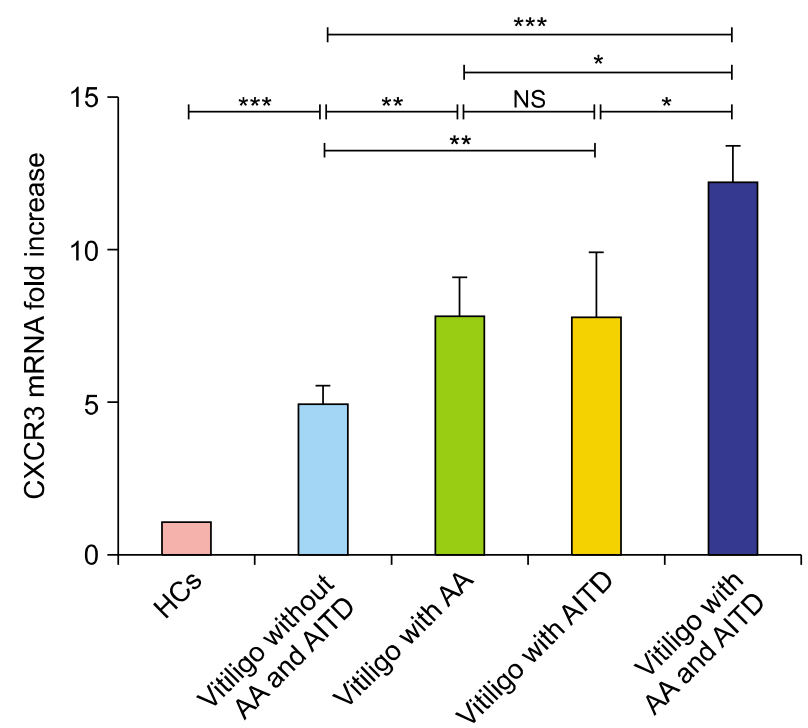

Fig. 4. The mRNA expression of CXCR3 in circulation. ANOVA test was applied. The mRNA expression of CXCR3 was significantly higher in patients with NSV than in healthy controls (HCs). In addition, it was higher in patients who had nonsegmental vitiligo (NSV) with either alopecia areata (AA) or autoimmune thyroid disease (AITD), and in those who had NSV with both AA and AITD than in patients who had NSV without AA or AITD. There was no significant difference in CXCR3 mRNA expression between patients who had NSV with AA and those who had NSV with AITD. NS: no significance. ${ }^{*} p<0.05$, ${ }^{* *} p<0.01,{ }^{* * *} p<0.001$. 
A

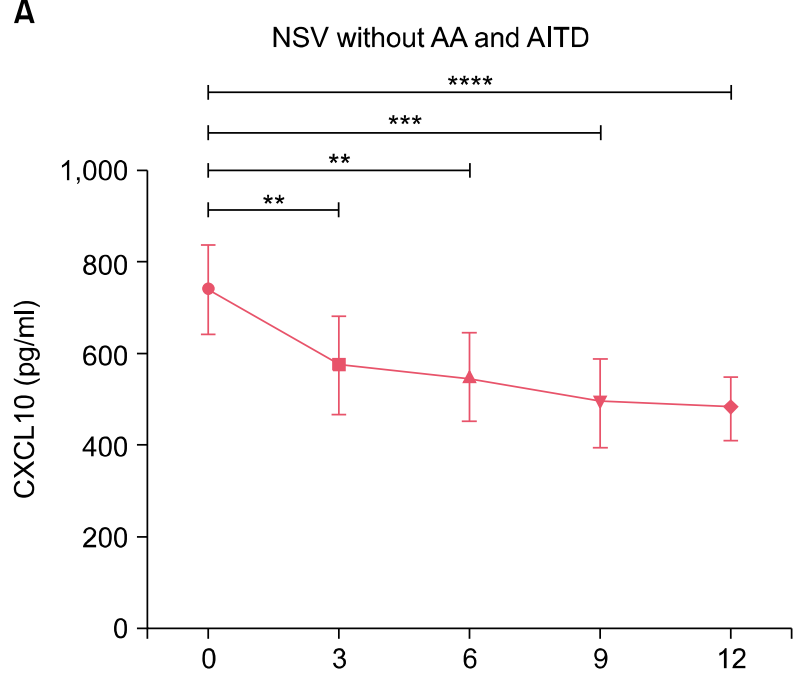

C

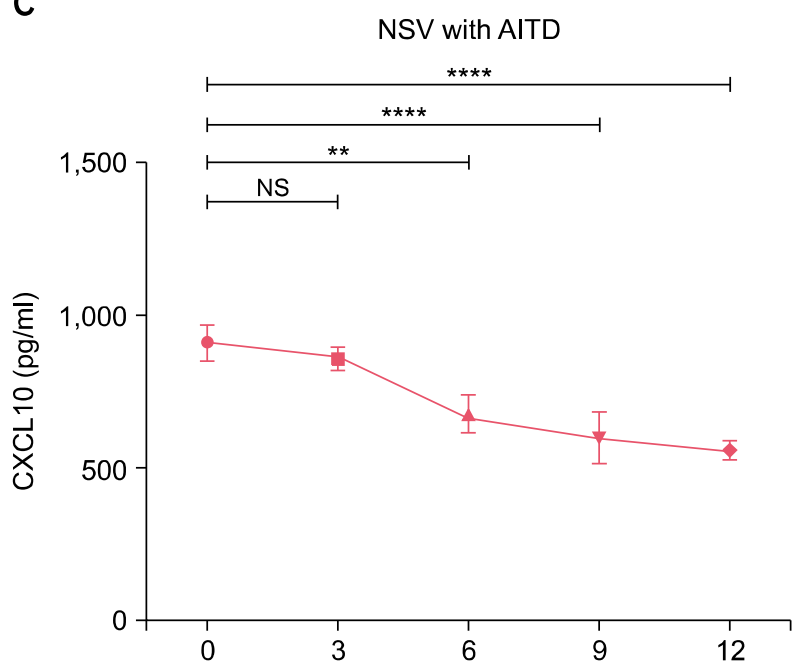

B

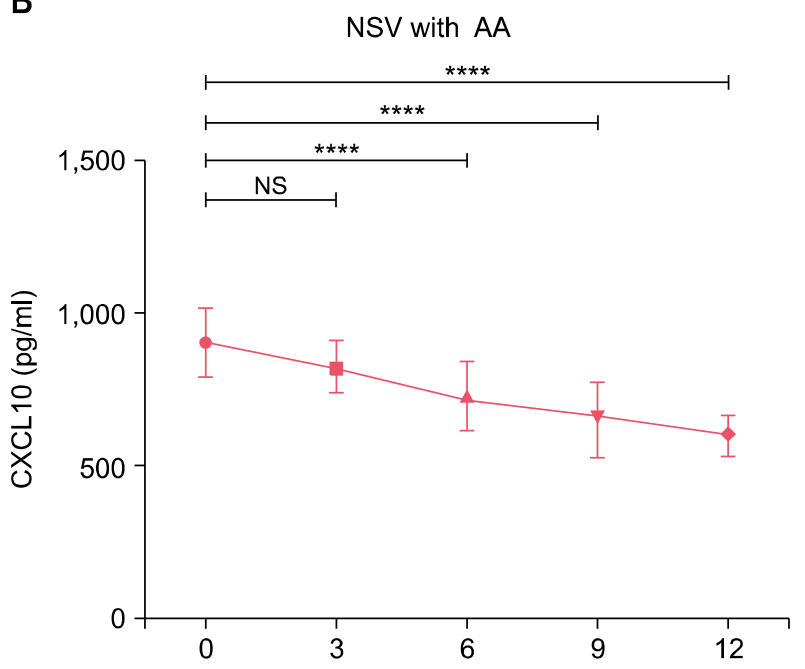

D

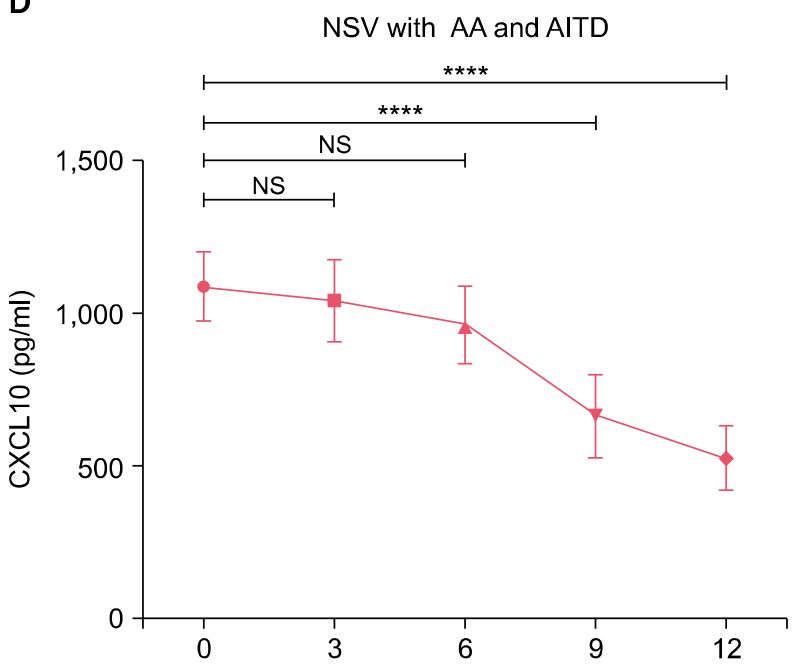

Fig. 5. Dynamic changes in CXCL10 levels after $0,3,6,9$, and 12 months of treatment. ANOVA test was applied. In patients who had non-segmental vitiligo (NSV) without alopecia areata (AA) or autoimmune thyroid disease (AITD) (A), the level of CXCL10 initially decreased significantly after 3 months treatment, then continuously decreased in month 6, 9, 12. In patients who had NSV with AA (B) and those who had NSV with AITD (C), the level of CXCL10 initially decreased significantly after 6 months of treatment, then continuously decreased in month 9, 12. In patients who had NSV with both AA and AITD (D), the level of CXCL10 initially decreased significantly after 9 months of treatment, then continuously decreased in month 12 . NS: no significance. ${ }^{* *} p<0.01,{ }^{* * *} p<$ $0.001, * * * * p<0.0001$.

NSV without AA or AITD had decreased significantly after 3 months ( $p<0.01$, Fig. 5), but no significant difference was observed between patients who had NSV with either AA or AITD and those who had NSV with both AA and AITD ( $p>0.05$ in both cases, Fig. 5). After 6 months, a significant decrease in CXCL10 levels had occurred in patients those who had NSV with AA or AITD alone $(p<$ 0.001 and $p<0.001$, respectively), but no significant difference was observed in patients who had NSV with both AA and AITD ( $p>0.05$, Fig. 5). After 9 months treatment, CXCL10 levels had decreased significantly in patients who had NSV with both AA and AITD ( $p<0.001$, Fig. 5). Lastly, after 12 months treatment, the mean level of CXCL10 of all the patients is lower than that after 9 months treatment. Moreover, the levels of CXCL10 in patients who had NSV with both AA and AITD were higher than in those who had NSV with AA or AITD alone in month 3 and 6. The levels of CXCL10 in patients who had NSV with either AA or AITD were higher than in those who had NSV without AA or AITD in month 3, 6, 9, and 12 (Fig. 6). 
A

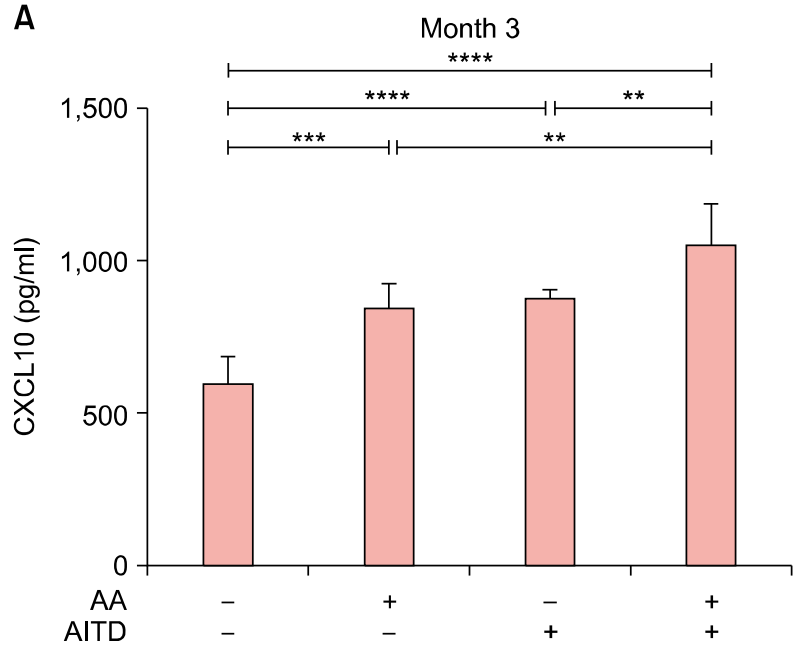

C

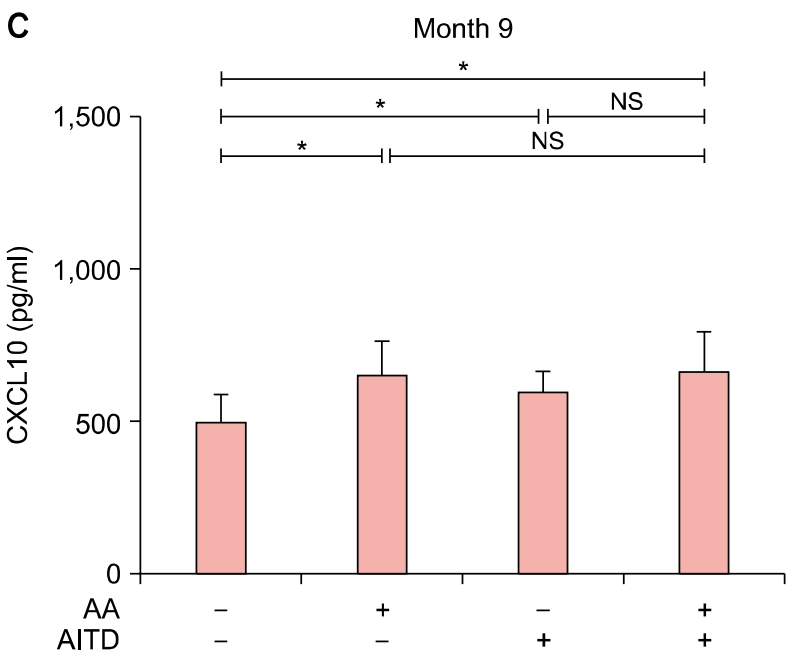

B
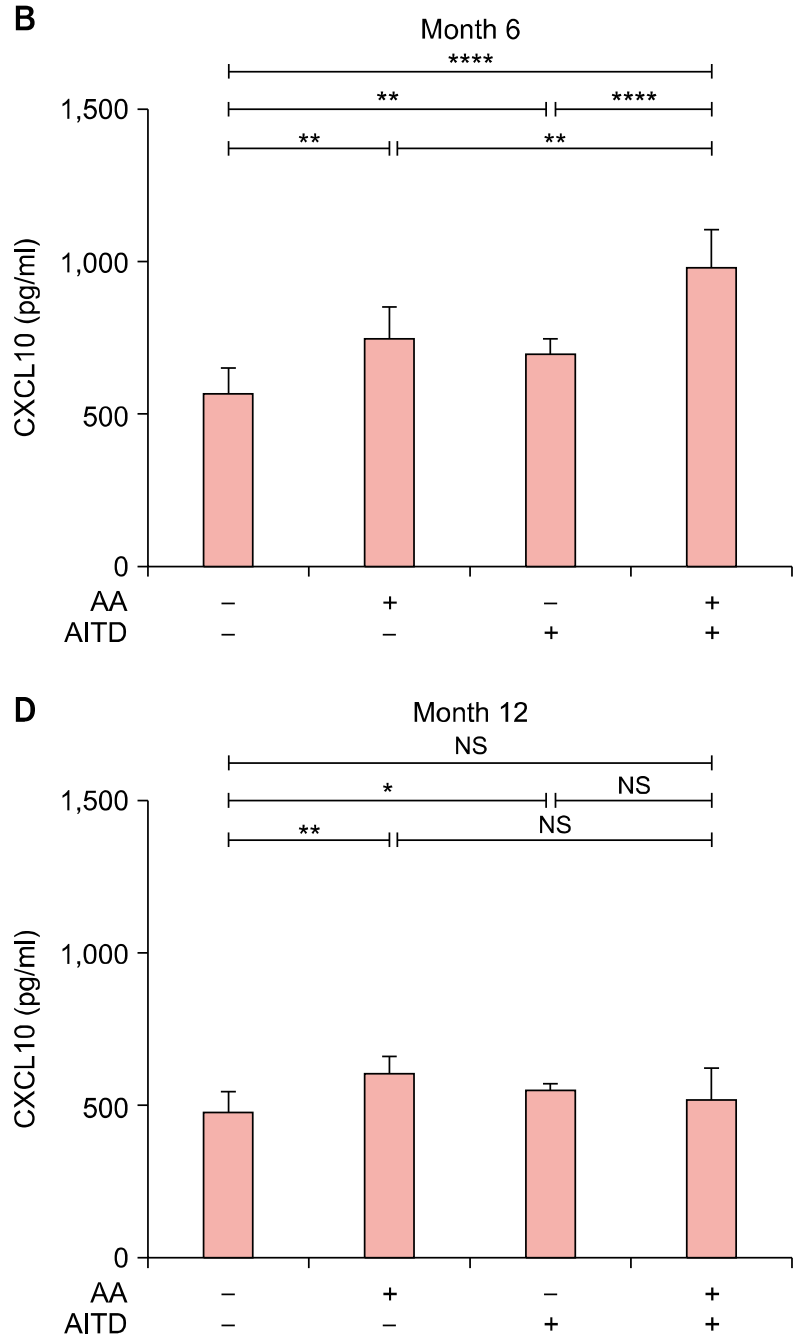

Fig. 6. Different levels of CXCL10 between groups in month 3, month 6, month 9, and month 12. ANOVA test was applied. The levels of CXCL10 in patients who had NSV with both alopecia areata (AA) and autoimmune thyroid disease (AITD) were higher than in those who had non-segmental vitiligo (NSV) with AA or AITD alone in month 3 (A) and 6 (B). The levels of CXCL10 in patients who had NSV with either AA or AITD were higher than in those who had NSV without AA or AITD in month 3 (A), 6 (B), 9 (C), and 12 (D). NS: no significance. ${ }^{*} p<0.05,{ }^{* *} p<0.01,{ }^{* * *} p<0.001,{ }^{* * * *} p<0.0001$.

\section{Grade $0 \quad \square$ Grade $1 \square$ Grade $2 \square$ Grade $3 \square$ Grade 4}

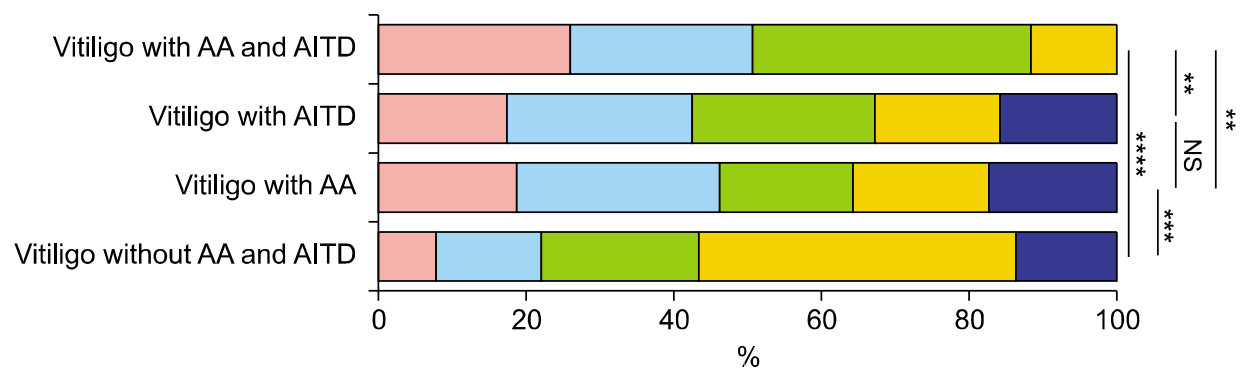

Fig. 7. The repigmentation scale in patients who had non-segmental vitiligo (NSV) with or without alopecia areata (AA) and autoimmune thyroid disease (AITD). ANOVA test was applied. Repigmentation was worse in patients who had NSV with both AA and AITD than in those who had NSV without either AA or AITD. Repigmentation was worse in patients who had NSV with either AA or AITD than in those who had NSV with AA and AITD. NS: no significance. ${ }^{* *} p<0.01,{ }^{* * *} p<0.001,{ }^{* * * *} p<0.0001$. 


\section{Poorer improvement in patients who had vitiligo with both AA and AITD}

In our follow-up, the repigmentation scores in patients who had NSV with both AA and AITD were lower than those in patients who had NSV without AA or AITD $(p<0.0001$ in both cases, Fig. 7). Patients who had NSV with both AA and AITD showed poorer repigmentation after 12 months than those who had NSV with AA or AITD alone $(p<0.01$ in all cases, Fig. 7). The repigmentation scores in patients who had NSV with either AA or AITD were poorer than those in patients who had NSV without AA or AITD $(p<$ 0.001 in both cases, Fig. 7).

\section{DISCUSSION}

Vitiligo is an autoimmune disease of the skin and several chemokines and other biomarkers were reported to be involved in its progression. Several studies have reported circulating markers that are associated with progressive vitiligo including chemokines and others. In the present study, the chemokines CXCL1, CXCL8, CXCL9, CXCL10, CXCL12, CXCL13, and CXCL16 were investigated in patients with NSV, and serum levels of CXCL9, CXCL10, CXCL12, and CXCL16 were found to be up-regulated in vitiligo. In addition, the serum levels of CXCL9, CXCL10, CXCL12, and CXCL16 were measured after treatment, and only the CXCL10 levels differed significantly after treatment.

CXCL9 and CXCL10 levels have been reported to be biomarkers for vitiligo progression. Maouia et al. ${ }^{21}$ reported that increased CXCL9 and CXCL10 levels were detected in patients with vitiligo compared with healthy controls, but the authors mentioned that difference of CXCL9 was less pronounced compared with the one observed for CXCL10. However, Rashighi et al. ${ }^{9}$ found elevated CXCL10 but not CLCX9 levels in patients with vitiligo. Richmond et al. ${ }^{22}$ showed the expression of both CXCL9 and CXCL10 correlated with disease activity in a mouse model of vitiligo, but only CXCL10 correlates with disease severity, which was consistent with our result. In addition, the patients with higher levels of CXCL10 before treatment still aggravated according to the VASI score after six months treatment while those with lower levels of CXCL10 showed no progression, which indicated the initial high level of CXCL10 before treatment was associated with the vitiligo progression. This part of study suggested serum CXCL10 could be used as a biomarker in vitiligo.

Vitiligo, AA and AITD are all autoimmune diseases, and striking similarities in pathogenesis have been identified at the level of both the innate and adaptive immune system.
Th1 cells produce IFN- $\gamma$, inducing the production of CXCL10, which can in turn recruit more Th1 cells ${ }^{23}$. CD8 + $\mathrm{T}$ cells may attack melanocytes directly during the onset and progression of vitiligo ${ }^{24,25}$. Notably, AITD is considered as a Th1-mediated disease, and the anti-thyroid antibodies that occur during AITD are more common in patients with vitiligo than in healthy controls ${ }^{17,26}$. The concurrence of depigmentation and hair loss in patients with vitiligo and $\mathrm{AA}$ implies that the two diseases share similar pathogeneses that depend on cytotoxic CD8 + T cells located close to melanocytes and hair follicles within the $\operatorname{skin}^{27,28}$. Studies in animal models of vitiligo and AA have specifically implicated an IFN- $\gamma$-related immune response, with IFN- $\gamma$, IFN- $\gamma$-induced chemokines, and cytotoxic CD8 $+\mathrm{T}$ cells as the main drivers of disease pathogenesis ${ }^{3}$. Epidemiologic study revealed AITD was the most common comorbidity in patients with vitiligo, with the prevalence up to $34 \%{ }^{29}$, with shared heritable susceptibility genes $^{30}$. What's more, melanocyte-specific antigen expression in thyroid tissue was reported in patients with Hashimoto's thyroiditis, as well as in healthy individuals ${ }^{31}$. Vitiligo concomitant with AITD is more common among female, and the disease tends to have a longer duration and to cover a higher body surface area ${ }^{32}$. Gey et al. ${ }^{32}$ reported a larger body surface area involvement was associated with the presence of AITD in 626 patients with vitiligo. Based on the above epidemiologic studies and mechanism studies, notice should be taken on the autoimmune comorbidity of vitiligo, especially AA and AITD. In euthyroid chronic AITD, especially in middle-aged female, the serum levels of CXCL10 are significantly higher than in healthy controls ${ }^{33}$. In the serum of patients with AA, CXCL10 levels have been found to be significantly higher than in healthy controls ${ }^{21}$. One study reported higher serum CXCL10 levels in patients with NSV and in those with NSV plus AITD than in healthy controls ${ }^{34}$. Based on our result of biomarker of CXCL10 and above reports, we further explored the association between vitiligo and its concomitant AA and AITD. To our knowledge, this is the first study to analyze serum CXCL10 levels among patients who had NSV with AA and AITD. Serum CXCL10 levels were higher in patients who had NSV with both AA and AITD than those who had NSV without either AA or AITD and those who had NSV with AA or AITD alone. The concurrence of vitiligo with AA and AITD may contribute to more active reaction in immunological mechanism. CXCR3 is a $\mathrm{G} \alpha$ i protein-coupled receptor in the CXC chemokine receptor family. CXCL10 belongs to the CXC subfamily chemokine and exerts its function by binding to CXCR3, which is highly expressed on CD4 $+\mathrm{T}$ cells and effector $\mathrm{CD} 8+\mathrm{T}_{\text {cells }}{ }^{35}$. In the present study, we showed that CXCR3 
mRNA levels in the PBMCs of patients who had NSV with both AA and AITD were higher than those of patients who had NSV without AA or AITD, corroborating the findings regarding serum $\mathrm{CXCL}^{10}$ levels. This result further confirmed the role of CXCL10 in concurrence of $\mathrm{AA}$ and AITD in patients with NSV. Our previous study have demonstrated serum CXCL10 might be a novel biomarker for monitoring disease activity and guiding treatment in progressive vitiligo ${ }^{10}$. However, the study did not distinguish the difference in vitiligo patients with and without the concomitance of AITD and AA. In this long-term prospective cohort study, the time to show a significant decrease of CXCL10 was 9 months after treatment in patients who had NSV with both AA and AITD, 6 months in patients who had NSV with AA or AITD alone, and 3 months in patients who had NSV without AA or AITD. Furthermore, after 12 months treatment, poorer repigmentation was observed in patients who had NSV with both AA and AITD than those who had NSV without AA or AITD. Collectively, these results further clarified the concurrence of vitiligo with AA and AITD might contribute to more active reaction in immunological mechanism.

There are several limitations of our study. The sample size is relatively small size, which limits the generalizability of the findings. Moreover, it is believed chemokine expression in vitiligo tissues is more important to evaluate disease progression in vitiligo. However, we did not collect the vitiligo tissues for chemokine expression analysis due to the difficulty for biopsy. More and larger clinical trials should be performed to further elucidate the serum CXCL10 as a prognostic marker for NSV concomitant with AITD and AA.

In summary, CXCL10 could be used as a biomarker to predict the future progression in NSV. CXCR3 might play a role in the mechanisms of vitiligo and its comorbidity AA and AITD. Moreover, considering that patients who had NSV with both AA and AITD showed poorer therapy outcome, dermatologists should take special notice of patients with NSV for the concomitance of AITD and AA.

\section{ACKNOWLEDGMENT}

This study was supported by the National Natural Science Foundation of China (Grant No. 81872544, No. 81803159, No. 81703145, No. 81703121, No. 81573064 and No. 81472901).

\section{CONFLICTS OF INTEREST}

The authors have nothing to disclose.

\section{ORCID}

Li Zhang, https://orcid.org/0000-0003-2259-0817

Xinya Xu, https://orcid.org/0000-0002-7600-6914

Shujun Chen, https://orcid.org/0000-0003-0999-5203

Yuli Kang, https://orcid.org/0000-0001-8131-1238

Xiuxiu Wang, https://orcid.org/0000-0002-6791-8524

Chengfeng Zhang, https://orcid.org/0000-0002-1302-5667

Leihong Xiang, https://orcid.org/0000-0002-6999-4686

\section{REFERENCES}

1. Picardo M, Dell'Anna ML, Ezzedine K, Hamzavi I, Harris JE, Parsad D, et al. Vitiligo. Nat Rev Dis Primers 2015;1: 15011.

2. Harris JE. Cellular stress and innate inflammation in organspecific autoimmunity: lessons learned from vitiligo. Immunol Rev 2016;269:11-25.

3. Rork JF, Rashighi M, Harris JE. Understanding autoimmunity of vitiligo and alopecia areata. Curr Opin Pediatr 2016;28: 463-469.

4. Liu M, Murphy E, Amerson EH. Rethinking screening for thyroid autoimmunity in vitiligo. J Am Acad Dermatol 2016; 75:1278-1280.

5. Mockenhaupt M, Peters F, Schwenk-Davoine I, Herouy $Y$, Schraufstätter I, Elsner $\mathrm{P}$, et al. Evidence of involvement of CXC-chemokines in proliferation of cultivated human melanocytes. Int J Mol Med 2003;12:597-601.

6. Miniati A, Weng Z, Zhang B, Therianou A, Vasiadi M, Nicolaidou $E$, et al. Stimulated human melanocytes express and release interleukin-8, which is inhibited by luteolin: relevance to early vitiligo. Clin Exp Dermatol 2014;39:5457.

7. Lee HT, Shiao YM, Wu TH, Chen WS, Hsu YH, Tsai SF, et al. Serum BLC/CXCL13 concentrations and renal expression of CXCL13/CXCR5 in patients with systemic lupus erythematosus and lupus nephritis. J Rheumatol 2010;37:45-52.

8. Wutte N, Kovacs G, Berghold A, Reiter H, Aberer W, Aberer E. CXCL13 and B-cell activating factor as putative biomarkers in systemic sclerosis. Br J Dermatol 2013;169: 723-725.

9. Rashighi M, Agarwal P, Richmond JM, Harris TH, Dresser $\mathrm{K}$, Su MW, et al. CXCL10 is critical for the progression and maintenance of depigmentation in a mouse model of vitiligo. Sci Transl Med 2014;6:223ra23.

10. Wang XX, Wang QQ, Wu JQ, Jiang $M$, Chen L, Zhang CF, et al. Increased expression of CXCR3 and its ligands in patients with vitiligo and CXCL10 as a potential clinical marker for vitiligo. Br J Dermatol 2016;174:1318-1326.

11. Speeckaert R, Voet S, Hoste E, van Geel N. S100B is a potential disease activity marker in nonsegmental vitiligo. J Invest Dermatol 2017;137:1445-1453.

12. Kim JY, Lee EJ, Seo J, Oh SH. Impact of high-mobility group box 1 on melanocytic survival and its involvement in the pathogenesis of vitiligo. $\mathrm{Br}$ J Dermatol 2017;176:15581568. 
13. Speeckaert R, Ongenae K, van Geel N. Alterations of CXCL12 in serum of patients with vitiligo. J Invest Dermatol 2017; 137:1586-1588.

14. Li S, Zhu G, Yang Y, Jian Z, Guo S, Dai W, et al. Oxidative stress drives $\mathrm{CD}^{+}{ }^{+}$T-cell skin trafficking in patients with vitiligo through $\mathrm{CXCL} 16$ upregulation by activating the unfolded protein response in keratinocytes. J Allergy Clin Immunol 2017;140:177-189.e9.

15. Stinco G, Buligan C, Grimaldi F, Valent F, Patrone P. Serological screening for autoimmune polyendocrine syndromes in patients with vitiligo. J Eur Acad Dermatol Venereol 2012;26:1041-1042.

16. Laberge G, Mailloux CM, Gowan K, Holland P, Bennett DC, Fain PR, et al. Early disease onset and increased risk of other autoimmune diseases in familial generalized vitiligo. Pigment Cell Res 2005;18:300-305.

17. Vrijman C, Kroon MW, Limpens J, Leeflang MM, Luiten $\mathrm{RM}$, van der Veen JP, et al. The prevalence of thyroid disease in patients with vitiligo: a systematic review. $\mathrm{Br}$ J Dermatol 2012;167:1224-1235.

18. Xing L, Dai Z, Jabbari A, Cerise JE, Higgins CA, Gong W, et al. Alopecia areata is driven by cytotoxic $\mathrm{T}$ lymphocytes and is reversed by JAK inhibition. Nat Med 2014;20:10431049.

19. Taïeb A, Picardo M; VETF Members. The definition and assessment of vitiligo: a consensus report of the Vitiligo European Task Force. Pigment Cell Res 2007;20:27-35.

20. Fröhlich E, Wahl R. Thyroid autoimmunity: role of antithyroid antibodies in thyroid and extra-thyroidal diseases. Front Immunol 2017;8:521.

21. Maouia A, Sormani L, Youssef M, Helal AN, Kassab A, Passeron T. Differential expression of CXCL9, CXCL10, and IFN $-\gamma$ in vitiligo and alopecia areata patients. Pigment Cell Melanoma Res 2017;30:259-261.

22. Richmond JM, Bangari DS, Essien KI, Currimbhoy SD, Groom JR, Pandya AG, et al. Keratinocyte-derived chemokines orchestrate T-cell positioning in the epidermis during vitiligo and may serve as biomarkers of disease. J Invest Dermatol 2017;137:350-358.

23. Campbell JD, Gangur V, Simons FE, HayGlass KT. Allergic humans are hyporesponsive to a CXCR3 ligand-mediated Th1 immunity-promoting loop. FASEB J 2004;18:329-331.

24. Lang KS, Caroli CC, Muhm A, Wernet D, Moris A, Schittek $B$, et al. HLA-A2 restricted, melanocyte-specific CD8(+) T lymphocytes detected in vitiligo patients are related to disease activity and are predominantly directed against MelanA/MART1. J Invest Dermatol 2001;116:891-897.

25. van den Boorn JG, Konijnenberg D, Dellemijn TA, van der Veen JP, Bos JD, Melief CJ, et al. Autoimmune destruction of skin melanocytes by perilesional $\mathrm{T}$ cells from vitiligo patients. J Invest Dermatol 2009;129:2220-2232.

26. Nanba $T$, Watanabe $M$, Inoue $N$, Iwatani $Y$. Increases of the Th1/Th2 cell ratio in severe Hashimoto's disease and in the proportion of Th17 cells in intractable Graves' disease. Thyroid 2009;19:495-501.

27. McElwee KJ, Gilhar A, Tobin DJ, Ramot Y, Sundberg JP, Nakamura $M$, et al. What causes alopecia areata? Exp Dermatol 2013;22:609-626.

28. Gilhar A, Paus R, Kalish RS. Lymphocytes, neuropeptides, and genes involved in alopecia areata. J Clin Invest 2007; 117:2019-2027.

29. Gill L, Zarbo A, Isedeh P, Jacobsen G, Lim HW, Hamzavi I. Comorbid autoimmune diseases in patients with vitiligo: a cross-sectional study. J Am Acad Dermatol 2016;74:295302.

30. Medici M, Porcu E, Pistis G, Teumer A, Brown SJ, Jensen $\mathrm{RA}$, et al. Identification of novel genetic Loci associated with thyroid peroxidase antibodies and clinical thyroid disease. PLoS Genet 2014;10:e1004123.

31. Spritz RA, Gowan K, Bennett DC, Fain PR. Novel vitiligo susceptibility loci on chromosomes 7 (AIS2) and 8 (AIS3), confirmation of SLEV1 on chromosome 17, and their roles in an autoimmune diathesis. Am J Hum Genet 2004;74: 188-191.

32. Gey A, Diallo A, Seneschal J, Léauté-Labrèze $C$, Boralevi $F$, Jouary $\mathrm{T}$, et al. Autoimmune thyroid disease in vitiligo: multivariate analysis indicates intricate pathomechanisms. Br J Dermatol 2013;168:756-761.

33. Rotondi M, Chiovato L, Romagnani S, Serio M, Romagnani $P$. Role of chemokines in endocrine autoimmune diseases. Endocr Rev 2007;28:492-520.

34. Ferrari SM, Fallahi P, Santaguida G, Virili C, Ruffilli I, Ragusa $F$, et al. Circulating CXCL10 is increased in nonsegmental vitiligo, in presence or absence of autoimmune thyroiditis. Autoimmun Rev 2017;16:946-950.

35. Van Raemdonck K, Van den Steen PE, Liekens S, Van Damme J, Struyf S. CXCR3 ligands in disease and therapy. Cytokine Growth Factor Rev 2015;26:311-327. 